\title{
Die Zukunft der Diabetologie ist digital und personalisiert
}

\author{
Die Digitalisierung wird die Medizin in revolutionärer \\ Weise verändern und dieser Prozess hat begonnen. \\ Auch in der Diabetologie bietet sie eine große Chance \\ auf eine nachhaltige Verbesserung der Patientenver- \\ sorgung. Die Verknüpfung und Nutzung von Daten \\ wird dazu beitragen, Zeit und Kosten zu sparen.
}

Die Versorgung bei Diabetes hat große Fortschritte gemacht. Viele Verbesserungen bei der Diabetestechnik und die Entwicklung innovativer Therapeutika haben Eingang in den Praxisalltag gefunden. „Doch trotz dieser Fortschritte bleiben die Ergebnisse der Diabetesversorgung hinter den Möglichkeiten zurück“, so Lars Kalfhaus, Geschäftsführer bei Roche Diabetes Care Deutschland. Es bestehe eine gewisse Diskrepanz zwischen den Ressourcen und den Behandlungsergebnissen. Trotz der vielen pharmakologischen und technischen Optionen erreiche nur eine Minderheit der Patienten das Behandlungsziel. So sind nur $65 \%$ der Diabetiker diagnostiziert, $42 \%$ kommen in den Genuss eines strukturierten Therapieprogramms, $26 \%$ erreichen ihre individuellen und $15 \%$ die leitliniengerechten Zielwerte. Dieser Unterversorgung stehen vielfältige Herausforderungen gegenüber: Die Patientenzahlen steigen, die Fachärzte werden weniger, die Budgets steigen nicht mehr und es werden ständig Therapieinnovationen entwickelt.

\section{Digitalisierung als Chance}

„Die Digitalsierung ist DIE Chance, es besser zu machen“, so Kalfhaus. Sie könne helfen, Therapieergebnisse zu verbessern, Zeit und Kosten zu sparen und somit wieder mehr Raum für das Wesentliche, nämlich den Patienten, zu schaffen. „Digitalisierung führt nicht zur Enthumanisierung der Medizin, im Gegenteil, sie wird zu einer Rehumanisierung führen“. Dass Digitalisierung und Datenvisualisierung die klinische Entscheidungsfindung erleichtern und verbessern, zeigt der Praxisalltag. Doch $78 \%$ der Diabetiker führen noch ein Handprotokoll, ob-

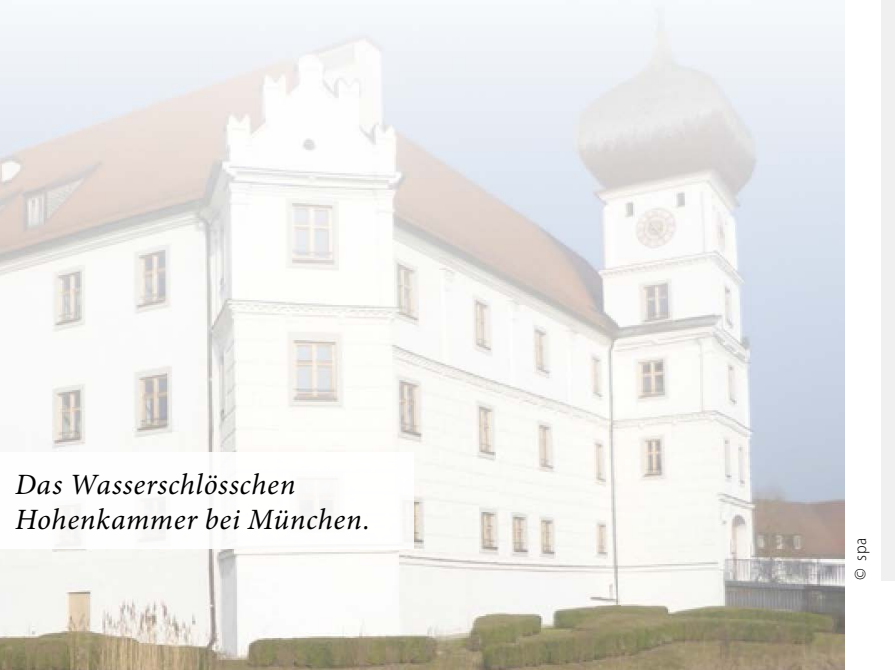

wohl bekannt ist, dass $60 \%$ der Einträge falsch sind. Aber $22 \%$ der Patienten laden schon Daten aus ihren Medizinprodukten hoch, was zur Zeitersparnis für den Arzt von $90 \%$ führt und in $79 \%$ die klinische Entscheidungsfindung verbessert. Mit den Handprotokollen ist Mustererkennung schwierig und zeitaufwendig, relevante Muster werden oft nicht erkannt. „Die automatischen Mustererkennungsalgorithmen sind dagegen $100 \%$ zuverlässig“, so Kalfhaus. Mit einer integrierten und personalisierten Versorgung könne eine $\mathrm{HbA}_{1 c}$-Senkung von $0,8 \%$ und eine Kostensenkung im ambulanten Bereich um $22 \%$ erreicht werden.

\section{Neues Denken gefragt}

An der kontinuierlichen Glukosemessung wird erkennbar, dass mehr Daten zwar mehr Optionen, aber auch Herausforderungen mit sich bringen. „Die Digitalsierung erfordert neues Denken und neue Lösungsansätze von allen“, so Kalfhaus. Diese müssen sich von der rückwärts gerichteten, auf einzelne Blutzuckermesspunkte konzentrierten Betrachtung auf eine vorausschauende, auf Kurven und Zusammenhänge gerichteten Sichtweise mit den daraus resultierenden Therapieempfehlungen umstellen. Es gehe nicht nur darum, einen guten Blutzuckerwert zu erreichen, sondern auch, dass der Patient mehr Zeit im Zielbereich verbringt. Der traditionelle Parameter $\mathrm{HbA}_{1 \mathrm{c}}$ habe eine deutlich geringere Aussagekraft, er sei viel weiter vom Patienten und seiner Lebenswirklichkeit entfernt. „In diesem Szenario wird sich die Rolle des Arztes zwar verändern, aber er wird keinesfalls überflüssig“, so Kalfhaus. Die Gestaltung der digitalen Zukunft brauche alle Versorgungsteilnehmer mit ihrer j Expertise. Dr. med. Peter Stiefelhagen

Quelle: Diabetes Mediendialog 2017, 30.3.-1.4.2017, Schloss Hohenkammer, Veranstalter: Roche

\section{Neue Ideen bei der IT-Entwicklung}

Das Roche Diabetes Care Design Co-Innovation Lab hat motivierte Typ1-Diabetiker zusammengebracht mit dem Ziel, innovative Konzepte unter Anwendung der schnellen und agilen Techniken des "Design Thinking" und des "Rapid Prototyping“ zu entwickeln. Danach wurden folgende Ideen von Kevin Röhl, Berlin, vorgestellt:

Chatbot: Darunter versteht man einen persönlichen Diabetesassistenten, der sich nahtlos in bereits bestehende Messenger wie Facebook oder WhatsApp integriert und damit übergreifend auf sämtlichen Geräten wie Smartphones, Smartwatches und Rechnern verfügbar ist. Das Bot ist ein intuitives Interface zu sämtlichen Datenbanken und kann beispielsweise die BE von einem Stück Pizza angeben oder die Frage beantworten, wie viele Teststreifen für den nächsten Urlaub benötigt werden.

GPS-Tracker: Damit können die GPS-Daten des Smartphones automatisch ausgelesen, mit den Blutzuckerwerten kombiniert und dann auf einer Karte im 3D Raum visualisiert werden. Damit wird dem Diabetiker geholfen, sich an bestimmte Ereignisse und Mahlzeiten zu erinnern. sti 